22

\title{
Геометрическая оптика во вращающемся диэлектрике
}

\author{
(ㄱ Н.Д. Миловский, А.О. Климин \\ Нижегородский государственный университет им. Н.И. Лобачевского, радиофизический фракультет, \\ 603950 Нижний Новгород, Россия \\ e-mail: mil1940@mail.ru
}

Поступила в редакцию 12.05.2020 г.

В окончательной редакции 17.07.2020 г.

Принята к публикации 25.08.2020 г.

\begin{abstract}
Вращающийся твердотельный однородный изотропный диэлектрик без дисперсии в сопровождающей вращение системе отсчета оказывается неоднородной анизотропной средой из-за воздействия двух конкурирующих физических механизмов: обусловленной вращением неоднородности свободного пространства и увлечения света движущейся средой. Во вращающемся диэлектрике в приближении геометрической оптики получены уравнение эйконала и соответствующая ему система обыкновенных дифференциальных уравнений в характеристической форме. Решениями уравнений при расчетных параметрах, определенных с помощью первых интегралов системы, являются пары „встречных“ $R$-траекторий лучей и $f$ - траекторий фазовых фронтов. Получена формула для интенсивности светового импульса, распространяющегося по произвольной $R$-траектории. „Встречные“ траектории обоих типов не имеют общих точек и смещены в противоположные стороны от прямой линии между конечными пунктами. Их структурные параметры (минимальное расстояние до оси вращения, длина дуги, область определения по азимутальной координате, оптическая длина и др.) изменяются под воздействием обоих физических механизмов, зависящих от скорости вращения. Замкнутые оптические пути в ВСО для ретрансляционной сети и для двухзеркального резонатора Фабри-Перо в генерирующем лазере можно создать с помощью двух типов адаптируемых к частоте вращения зеркал, у которых нормали к отражающим поверхностям должны иметь определенные разные углы с ортами лучевых векторов и волновых фронтов приходящего на рефлектор излучения. Эффект Саньяка является следствием неоднородности (деформации) свободного пространства по азимутальной координате, а его величина результат конкурирующего воздействия обоих физических механизмов.
\end{abstract}

Ключевые слова: твердотельный однородный изотропный диэлектрик, инерциальная и вращающаяся системы отсчета, геометрическая оптика, эйконал, $R$-траектории лучей и $f$-траектории фазовых фронтов, кольцевой вращающийся интерферометр (резонатор), эффект Саньяка.

DOI: $10.21883 /$ OS.2020.12.50335.150-20

\section{1. Введение}

Исследования в электродинамике движущихся сплошных сред можно разделить на два направления, каждое из которых по существу инициировано одним замечательным эффектом. Эффект увлечения света движущейся средой, экспериментально полученный (1851 г.) Физо, дал толчок развитию теоретических исследований, которые основаны на уравнениях Максвелла и материальных уравнениях Минковского, записанных в инерциальной (покоящейся или равномерно движущейся) системе отсчета (далее ИСО). В настоящее время такие разработки в наиболее совершенном виде систематической теории электродинамики сред с пространственно неоднородной скоростью движения $\mathbf{v}(\mathbf{r})$ в приближении первого порядка по отношению $|\mathbf{v}|$ к с (скорости света в вакууме) представлены в цикле замечательных работ группы Н.Н. Розанова [1,2]. Полученные в ИСО теоретические результаты в виде различных эффектов доступны для регистрации и измерений только наблюдателям (экспериментаторам), также находящимся в ИСО.

Однако не все оптические эффекты в движущейся среде целесообразно исследовать в ИСО. Так, после появления в начале прошлого века работы Саньяка [3], в которой была зарегистрирована разность фаз двух встречных волн на замкнутом оптическом пути внутри вращающегося кольцевого резонатора (далее КР), стало понятно, что адекватная интерпретация подобных экспериментов возможна только в сопровождающей вращение неинерциальной системе отсчета (далее $\mathrm{BCO})$. Возник интерес к распространению излучения во вращающемся диэлектрике, и появилась проблема корректного представления уравнений электродинамики в диэлектрической среде в ВСО, где покоящаяся среда, находясь под воздействием обусловленной вращением массовой центробежной силы, приобретает иные по сравнению с известными в ИСО диэлектрическую $\bar{\varepsilon}$ и магнитную $\bar{\mu}$ проницаемости. Эффект Саньяка в виде разности $\Delta \omega_{q}$ резонансных частот $\omega_{q}^{ \pm}$нормальных колебаний КР оказался востребованным для практического применения в гироскопии, где к настоящему времени созданы лазерные и волоконно-оптические гироскопы (кольцевые интерферометры с лазерными источниками света), которые используются в качестве датчиков угловой скорости, угла поворота и ориентации в пространстве вращающихся объектов. Поэтому эффект экспери- 
ментально изучался и теоретически интерпретировался многими исследователями, опубликовавшими большое число работ [4-8].

Для теоретического анализа экспериментальных результатов применялись уравнения электродинамики [9-11], основанные либо на некорректных преобразованиях электромагнитных полей и индукций из ИСО в ВСО, либо на феноменологически введенных постулатах, позволяющих не учитывать различие проницаемостей неподвижного диэлектрика в разных системах отсчета. Однако в подавляющем числе случаев точности расчетов $\Delta \omega_{q}$ (с учетом малой скорости вращения точки на траектории в КР и погрешностей измерения) было достаточно для установления его соответствия экспериментальному значению $\Delta \omega_{q}$. Поэтому поле научных исследований в ВСО оказалось сконцентрированным на изучении во вращающейся среде лишь эффекта Саньяка. В качестве исключения можно указать лишь работы [9,10]: в [9] рассматривалось наличие связей между TE- и ТМ-типами колебаний во вращающемся цилиндрическом резонаторе, а в [10] - распространение волн с разными ортогональными поляризациями поля по круговым встречным траекториям. В стороне от внимания ученых оказались практически все вопросы, которые в ИСО детально изучаются в таких разделах теоретической физики, как теория электромагнитного поля и электродинамика сплошных сред. В настоящее время появилась возможность для частичной ликвидации указанного пробела, поскольку в [12] получена полная система корректных уравнений электродинамики во вращающейся твердотельной среде, диэлектрическая $\bar{\varepsilon}$ и магнитная $\bar{\mu}$ проницаемости которой являются известными функциями цилиндрических координат ВСО. Новые явления, которые могут быть описаны в рамках этих уравнений, доступны для наблюдателей, находящихся в BCO.

Целью настоящей работы является изучение в приближении геометрической оптики (ГО) некоторых закономерностей поведения лучей и волновых фронтов электромагнитного монохроматического излучения во вращающемся твердотельном диэлектрике, который в ИСО является однородной изотропной средой без дисперсии с показателем преломления $n=\sqrt{\varepsilon \mu}$.

\section{2. Вывод уравнения эйконала во вращающейся диэлектрической среде}

Воспользуемся системой восьми скалярных уравнений электродинамики [12], которые описывают электромагнитное поле во вращающейся диэлектрической среде в сопровождающей вращение цилиндрической системе координат (ЦСК):

$$
\frac{\partial}{c \partial t}\left[\rho \gamma\left(\bar{B}_{\xi}-\beta \bar{E}_{\rho}\right)\right]+\frac{\partial}{\partial \rho}\left[\rho \bar{E}_{\psi}\right]-\frac{\partial}{\partial \psi}\left[\bar{E}_{\rho} / \gamma\right]=0
$$

$$
\begin{gathered}
\frac{\partial}{c \partial t} \bar{B}_{\psi}-\frac{\partial}{\partial \rho}\left[\bar{E}_{\xi} / \gamma\right]+\frac{\partial}{\partial \xi}\left[\bar{E}_{\rho} / \gamma\right]=0 \\
-\frac{\partial}{c \partial t}\left[\rho \gamma\left(\bar{B}_{\rho}+\beta \bar{E}_{\xi}\right)\right]-\frac{\partial}{\partial \psi}\left[\bar{E}_{\xi} / \gamma\right]+\frac{\partial}{\partial \xi}\left[\rho \bar{E}_{\psi}\right]=0 \\
\frac{\partial}{\partial \rho}\left[\rho \gamma\left(\bar{B}_{\rho}+\beta \bar{E}_{\xi}\right)\right]+\frac{\partial}{\partial \psi} \bar{B}_{\psi}+\frac{\partial}{\partial \xi}\left[\rho \gamma\left(\bar{B}_{\xi}-\beta \bar{E}_{\rho}\right)\right]=0 \\
\frac{\partial}{\partial \rho}\left[\frac{\rho \gamma}{\bar{\mu}}\left(\bar{n}^{2} \bar{E}_{\rho}-\beta \bar{B}_{\xi}\right)\right]+\frac{\partial}{\partial \psi}\left[\frac{\bar{n}^{2}}{\bar{\mu}} \bar{E}_{\psi}\right] \\
+\frac{\partial}{\partial \xi}\left[\frac{\rho \gamma}{\bar{\mu}}\left(\bar{n}^{2} \bar{E}_{\xi}+\beta \bar{B}_{\rho}\right)\right]=0 ; \\
\frac{\partial}{c \partial t}\left[\frac{\rho \gamma}{\bar{\mu}}\left(\bar{n}^{2} \bar{E}_{\rho}-\beta \bar{B}_{\xi}\right)\right]-\frac{\partial}{\partial \psi}\left[\frac{1}{\bar{\mu} \gamma} \bar{B}_{\xi}\right]+\frac{\partial}{\partial \xi}\left[\frac{\rho}{\bar{\mu}} \bar{B}_{\psi}\right]=0 \\
\frac{\partial}{c \partial t}\left[\frac{\bar{n}^{2}}{\bar{\mu}^{\mu}} \bar{E}_{\psi}\right]+\frac{\partial}{\partial \rho}\left[\frac{1}{\bar{\mu} \gamma} \bar{B}_{\xi}\right]-\frac{\partial}{\partial \xi}\left[\frac{1}{\bar{\mu} \gamma} \bar{B}_{\rho}\right]=0 ; \\
\frac{\partial}{c \partial t}\left[\frac{\rho \gamma}{\bar{\mu}}\left(\bar{n}^{2} \bar{E}_{\xi}+\beta \bar{B}_{\rho}\right)\right]-\frac{\partial}{\partial \rho}\left[\frac{\rho}{\bar{\mu}} \bar{B}_{\psi}\right]+\frac{\partial}{\partial \psi}\left[\frac{1}{\bar{\mu} \gamma} \bar{B}_{\rho}\right]=0 .
\end{gathered}
$$

В (1) использованы зависящие от координат $\rho, \psi, \xi$ проекции $\left(\bar{E}_{\delta}, \bar{B}_{\delta}\right)$ напряженности электрического поля $\overline{\mathbf{E}}$ и индукции магнитного поля $\overline{\mathbf{B}}$ на оси ЦСК, диэлектрическая $\bar{\varepsilon}$ и магнитная $\bar{\mu}$ проницаемости среды $\left(\bar{n}^{2}=\overline{\varepsilon \mu}\right)$, нормированный на скорость света $c$ вектор скорости вращения $\boldsymbol{\beta}=(\Omega \rho / c) \boldsymbol{\psi}_{0} \equiv K \rho \boldsymbol{\psi}_{0} \equiv \beta \boldsymbol{\psi}_{0}$ точки вещества, находящейся на расстоянии $\rho$ от оси, и фактор $\gamma=1 / \sqrt{1-\beta^{2}}$, который определяет обусловленную вращением неоднородность свободного пространства. Зависимости $\bar{\varepsilon}$ и $\bar{n}^{2}$ от координат ВСО можно считать известными, если в ИСО определены $\varepsilon, n^{2}$ и если известны упругие и электрострикционные свойства твердотельного материала. В случае жидкой (или газообразной) среды, находящейся, например, в пространстве между двумя вращающимися соосными цилиндрами, для установления зависимостей $\bar{\varepsilon}$ и $\bar{n}^{2}$ от координат помимо электрострикционных свойств необходимо знать плотность, вязкость и сжимаемость жидкости для определения радиального профиля потока плотности импульса вращающегося вещества [13].

Уравнения (1) можно записать в векторной форме, используя операции дивергенции и ротора в ЦСК и векторы

$$
\begin{gathered}
\overline{\mathbf{B}}_{\perp}+\overline{\mathbf{B}}_{\|} \equiv\left(\bar{B}_{\rho} \boldsymbol{\rho}_{0}+\bar{B}_{\xi} \xi_{0}\right)+\bar{B}_{\psi} \boldsymbol{\psi}_{0}=\overline{\mathbf{B}} \\
\overline{\mathbf{E}}_{\perp}+\overline{\mathbf{E}}_{\|} \equiv\left(\bar{E}_{\rho} \boldsymbol{\rho}_{0}+\bar{E}_{\zeta} \xi_{0}\right)+\bar{E}_{\psi} \boldsymbol{\psi}_{0}=\overline{\mathbf{E}},
\end{gathered}
$$

ориентированные перпендикулярно и параллельно $\boldsymbol{\beta}$. В новой записи первые три уравнения (1) приобретают смысл проекций на оси ЦСК векторного уравнения

$$
\operatorname{rot}\left(\overline{\mathbf{E}}_{\|}+\frac{1}{\gamma} \overline{\mathbf{E}}_{\perp}\right)+\frac{1}{c} \frac{\partial}{\partial t}\left\{\overline{\mathbf{B}}_{\|}+\gamma\left(\overline{\mathbf{B}}_{\perp}+\left[\boldsymbol{\beta} \times \overline{\mathbf{E}}_{\perp}\right]\right)\right\}=0,
$$

четвертое уравнение (1) принимает форму

$$
\operatorname{div}\left\{\overline{\mathbf{B}}_{\|}+\gamma\left(\overline{\mathbf{B}}_{\perp}+\left[\boldsymbol{\beta} \times \overline{\mathbf{E}}_{\perp}\right]\right)\right\}=0,
$$


пятое уравнение (1) с учетом локальных материальных связей $(\overline{\mathbf{B}}=\bar{\mu} \overline{\mathbf{H}}, \overline{\mathbf{D}}=\bar{\varepsilon} \overline{\mathbf{E}})$ трансформируется в скалярное уравнение

$$
\begin{aligned}
& \operatorname{div}\left\{\overline{\boldsymbol{\varepsilon}}\left(\overline{\mathbf{E}}_{\|}+\gamma \overline{\mathbf{E}}_{\perp}\right)-(\gamma / \bar{\mu})\left[\boldsymbol{\beta} \times \overline{\mathbf{B}}_{\perp}\right]\right\} \\
& \equiv \operatorname{div}\left\{\overline{\mathbf{D}}_{\|}+\gamma\left(\overline{\mathbf{D}}_{\perp}-\left[\boldsymbol{\beta} \times \overline{\mathbf{H}}_{\perp}\right]\right)\right\}=0,
\end{aligned}
$$

и, наконец, три последних уравнения (1) преобразуются в векторное уравнение

$$
\begin{aligned}
& \operatorname{rot}\left\{\left(\overline{\mathbf{B}}_{\|}+\frac{1}{\gamma} \overline{\mathbf{B}}_{\perp}\right) \frac{1}{\bar{\mu}}\right\} \\
& -\frac{1}{c} \frac{\partial}{\partial t}\left\{\bar{\varepsilon}\left(\overline{\mathbf{E}}_{\|}+\gamma \cdot \overline{\mathbf{E}}_{\perp}\right)-(\gamma / \bar{\mu})\left[\boldsymbol{\beta} \times \overline{\mathbf{B}}_{\perp}\right]\right\} \\
& \equiv \operatorname{rot}\left(\overline{\mathbf{H}}_{\|}+\frac{1}{\gamma} \overline{\mathbf{H}}_{\perp}\right) \\
& -\frac{1}{c} \frac{\partial}{\partial t}\left\{\overline{\mathbf{D}}_{\|}+\gamma\left(\overline{\mathbf{D}}_{\perp}-\left[\boldsymbol{\beta} \times \overline{\mathbf{H}}_{\perp}\right]\right)\right\} \equiv 0 .
\end{aligned}
$$

В отсутствие вращения $(\boldsymbol{\beta}=0)$ с учетом замен обозначений полей $(\bar{V} \rightarrow V)$ и параметров диэлектрика $(\bar{\varepsilon} \rightarrow \varepsilon ; \bar{\mu} \rightarrow \mu)$ уравнения (3) переходят в уравнения Максвелла для покоящейся однородной изотропной среды $(\mathbf{B}=\mu \mathbf{H}, \mathbf{D}=\varepsilon \mathbf{E})$ без источников в ИСО:

$$
\operatorname{rot} \mathbf{E}+c^{-1}(\partial \mathbf{B} / \partial t)=0
$$

$\operatorname{div} \mathbf{B}=0 ; \operatorname{div} \mathbf{D}=0 ; \operatorname{rot} \mathbf{H}-c^{-1}(\partial \mathbf{D} / \partial t)=0$.

Монохроматическое решение уравнений (3) можно искать в виде произведений

$$
\begin{aligned}
& \left(\overline{\mathbf{B}}_{\perp}, \overline{\mathbf{B}}_{\|}, \overline{\mathbf{E}}_{\perp}, \overline{\mathbf{E}}_{\|}\right)=\operatorname{Re}\left\{\left(\tilde{\mathbf{B}}_{\perp}^{0}, \tilde{\mathbf{B}}_{\|}^{0}, \tilde{\mathbf{E}}_{\perp}^{0}, \tilde{\mathbf{E}}_{\|}^{0}\right)\right. \\
& \times \exp [i \omega t-i(\omega / c) \Phi(\rho, \psi, \zeta)]\}
\end{aligned}
$$

медленно меняющихся на пространственном периоде $\lambda=c / \omega$ векторов $\left(\tilde{\mathbf{B}}_{\perp}^{0}, \tilde{\mathbf{B}}_{\|}^{0}, \tilde{\mathbf{E}}_{\perp}^{0}, \tilde{\mathbf{E}}_{\|}^{0}\right)$ и быстро осциллирующей функции $\exp [-i(\omega / c) \Phi(\rho, \psi, \xi)]$, в которой эйконал $\Phi$ также медленно изменяется на длине $\lambda$. Подставляя (4) в (3), удается получить в нулевом порядке геометрооптического приближения систему однородных уравнений

$$
\begin{array}{r}
-\left[\nabla \boldsymbol{\Phi} \times\left(\tilde{\mathbf{E}}_{\|}^{0}+\gamma^{-1} \tilde{\mathbf{E}}_{\perp}^{0}\right)\right]+\left\{\tilde{\mathbf{B}}_{\perp}^{0}+\gamma\left(\tilde{\mathbf{B}}_{\perp}^{0}+\left[\boldsymbol{\beta} \times \tilde{\mathbf{E}}_{\perp}^{0}\right]\right)\right\}=0 ; \\
\left(\nabla \boldsymbol{\Phi} \cdot\left\{\tilde{\mathbf{B}}_{\|}^{0}+\gamma\left(\tilde{\mathbf{B}}_{\perp}^{0}+\left[\boldsymbol{\beta} \times \tilde{\mathbf{E}}_{\perp}^{0}\right]\right)\right\}\right)=0 ; \\
\left(\nabla \boldsymbol{\Phi} \cdot\left\{\bar{\varepsilon}\left(\tilde{\mathbf{E}}_{\|}^{0}+\gamma \tilde{\mathbf{E}}_{\perp}^{0}\right)-(\gamma / \bar{\mu})\left[\boldsymbol{\beta} \times \tilde{\mathbf{B}}_{\perp}^{0}\right]\right\}\right)=0 ; \\
{\left[\nabla \boldsymbol{\Phi} \times\left\{\left(\tilde{\mathbf{B}}_{\|}^{0}+\gamma^{-1} \tilde{\mathbf{B}}_{\perp}^{0}\right) / \bar{\mu}\right\}\right]+\left\{\bar{\varepsilon}\left(\tilde{\mathbf{E}}_{\|}^{0}+\gamma \tilde{\mathbf{E}}_{\perp}^{0}\right)\right.} \\
\left.-(\gamma / \bar{\mu})\left[\boldsymbol{\beta} \times \tilde{\mathbf{B}}_{\perp}^{0}\right]\right\} \equiv 0
\end{array}
$$

относительно векторных комплексных амплитуд $\left(\tilde{\mathbf{B}}_{\perp}^{0}, \tilde{\mathbf{B}}_{\|}^{0}, \tilde{\mathbf{E}}_{\perp}^{0}, \tilde{\mathbf{E}}_{\|}^{0},\right)$, в которой вектор

$$
\nabla \boldsymbol{\Phi}=\frac{\partial \Phi}{\partial \rho} \boldsymbol{\rho}_{0}+\frac{1}{\rho} \frac{\partial \Phi}{\partial \psi} \boldsymbol{\psi}_{0}+\frac{\partial \Phi}{\partial \xi} \xi_{0} \equiv|\nabla \boldsymbol{\Phi}| \mathbf{n}_{0}
$$

определен во вращающейся ЦСК. Уравнения (5) представляют собой систему восьми однородных скалярных уравнений

$$
\begin{aligned}
& \gamma \tilde{B}_{\rho}^{0}+\frac{\partial \Phi}{\partial \xi} \tilde{E}_{\psi}^{0}+\left(\gamma \beta-\frac{1}{\gamma \rho} \frac{\partial \Phi}{\partial \psi}\right) \tilde{E}_{\xi}^{0}=0 \\
& \tilde{B}_{\psi}^{0}-\frac{1}{\gamma} \frac{\partial \Phi}{\partial \xi} \tilde{E}_{\rho}^{0}+\left(\frac{1}{\gamma} \frac{\partial \Phi}{\partial \rho}\right) \tilde{E}_{\xi}^{0}=0 ; \\
& \gamma \tilde{B}_{\xi}^{0}+\left(\frac{1}{\gamma \rho} \frac{\partial \Phi}{\partial \psi}-\gamma \beta\right) \tilde{E}_{\rho}^{0}-\frac{\partial \Phi}{\partial \rho} \tilde{E}_{\psi}^{0}=0 ; \\
& \gamma \frac{\partial \Phi}{\partial \rho} \tilde{B}_{\rho}^{0}+\frac{1}{\rho} \frac{\partial \Phi}{\partial \psi} \tilde{B}_{\psi}^{0}+\gamma \frac{\partial \Phi}{\partial \xi} \tilde{B}_{\xi}^{0} \\
& -\gamma \frac{\partial \Phi}{\partial \xi} \beta \tilde{E}_{\rho}^{0}+\gamma \frac{\partial \Phi}{\partial \rho} \beta \tilde{E}_{\xi}^{0}=0 \\
& \frac{\gamma}{\bar{\mu}} \beta \frac{\partial \Phi}{\partial \xi} \tilde{B}_{\rho}^{0}-\frac{\gamma}{\bar{\mu}} \beta \frac{\partial \Phi}{\partial \rho} \tilde{B}_{\xi}^{0}+\bar{\varepsilon} \gamma \frac{\partial \Phi}{\partial \rho} \tilde{E}_{\rho}^{0} \\
& +\bar{\varepsilon} \frac{1}{\rho} \frac{\partial \Phi}{\partial \psi} \tilde{E}_{\psi}^{0}+\bar{\varepsilon} \gamma \frac{\partial \Phi}{\partial \xi} \tilde{E}_{\xi}^{0}=0 ; \\
& -\frac{1}{\bar{\mu}} \frac{\partial \Phi}{\partial \xi} \tilde{B}_{\psi}^{0}+\frac{\gamma}{\bar{\mu}}\left(\frac{1}{\gamma^{2} \rho} \frac{\partial \Phi}{\partial \psi}-\beta\right) \tilde{B}_{\xi}^{0}+\bar{\varepsilon} \gamma \tilde{E}_{\rho}^{0}=0 \\
& \frac{1}{\gamma \bar{\mu}} \frac{\partial \Phi}{\partial \xi} \tilde{B}_{\rho}^{0}-\frac{1}{\gamma \bar{\mu}} \frac{\partial \Phi}{\partial \rho} \tilde{B}_{\xi}^{0}+\bar{\varepsilon} \tilde{E}_{\psi}^{0}=0 ; \\
& \frac{\gamma}{\bar{\mu}}\left(\beta-\frac{1}{\gamma^{2} \rho} \frac{\partial \Phi}{\partial \psi}\right) \tilde{B}_{\rho}^{0}+\frac{1}{\mu} \frac{\partial \Phi}{\partial \rho} \tilde{B}_{\psi}^{0}+\bar{\varepsilon} \gamma \tilde{E}_{\xi}^{0}=0
\end{aligned}
$$

относительно шести проекций $\tilde{E}_{\delta}^{0}, \quad \tilde{B}_{\delta}^{0} \quad$ векторов $\left(\tilde{\mathbf{B}}_{\perp}^{0}, \tilde{\mathbf{B}}_{\|}^{0}, \tilde{\mathbf{E}}_{\perp}^{0}, \tilde{\mathbf{E}}_{\|}^{0}\right)$ на оси ЦСК. Последовательно исключая из уравнений (7) неизвестные $\left(\tilde{B}_{\rho}^{0}, \tilde{B}_{\psi}^{0}, \tilde{E}_{\psi}^{0}, \tilde{E}_{\rho}^{0}\right)$ и используя условия нетривиальности их решений $\left(\tilde{E}_{\xi}^{0} \neq 0\right.$ и $\left.\tilde{B}_{\xi}^{0} \neq 0\right)$, можно получить два одинаковых уравнения эйконала

$$
\begin{aligned}
& \left(\frac{\partial \Phi}{\partial \rho}\right)^{2}+\left(\frac{\partial \Phi}{\partial \xi}\right)^{2}+\left(\frac{1}{\rho} \frac{\partial \Phi}{\partial \psi}\right)^{2}-\frac{\bar{n}^{2}-1}{1-\beta^{2}} \\
& -\left(1+K \frac{\partial \Phi}{\partial \psi}\right)^{2}=0
\end{aligned}
$$

Решения (8) применимы для описания распространения ТЕ- и ТМ-типов волн во вращающейся среде $\left.\left(\bar{n}^{2} \neq 0\right)\right)$, включая вакуум $(\bar{n} \equiv \hat{n}=1)$, где

$$
\left(\frac{\partial \Phi}{\partial \rho}\right)^{2}+\left(\frac{\partial \Phi}{\partial \xi}\right)^{2}+\left(\frac{1}{\rho} \frac{\partial \Phi}{\partial \psi}\right)^{2}-\left(1+K \frac{\partial \Phi}{\partial \psi}\right)^{2}=0
$$

В случае $K=0$, когда отсутствует неоднородность пространства по координатам $\rho$ и $\psi$, а также изменения плотности и показателя преломления среды диэлектрика $(\bar{n} \rightarrow n)$, уравнение $(8)$ преобразуется в очевидную для ИСО форму

$$
\left(\frac{\partial \Phi_{0}}{\partial \rho}\right)^{2}+\left(\frac{\partial \Phi_{0}}{\partial z}\right)^{2}+\left(\frac{1}{\rho} \frac{\partial \Phi_{0}}{\partial \theta}\right)^{2}-n^{2}=0
$$




\section{3. Система дифференциальных уравнений в характеристической форме}

Нелинейное уравнение в частных производных первого порядка (8) в соответствии с общей теорией решения подобных уравнений класса Гамильтона-Якоби $[14,15]$ следует преобразовать в функционал

$$
\begin{aligned}
& P^{2}+Z^{2}+\left(\frac{Q}{\beta}\right)^{2}-(1+Q)^{2}-\frac{\bar{n}^{2}-1}{1-\beta^{2}} \\
& \equiv H\left(x_{1}, x_{1}, x_{2}, x_{3}, p_{1}, p_{2}, p_{3}\right)=0,
\end{aligned}
$$

в котором координаты $x_{1}=\rho=\beta / K, x_{2}=\psi ; x_{3}=\xi$ и соответствующие им компоненты градиента эйконала (или „импульсы“ [16])

$$
\begin{gathered}
p_{1}=(\partial \Phi / \partial \rho) \equiv P, p_{2}=(\partial \Phi / \partial \psi) \equiv Q / K, \\
p_{3}=(\partial \Phi / \partial \xi) \equiv Z,
\end{gathered}
$$

рассматриваются как шесть независимых переменных аргументов. Это позволяет получить решение $\Phi(\rho, \psi, \xi)$ уравнения (8) через решения $\rho=\rho(\tau), \quad \psi=\psi(\tau)$, $\xi=\zeta(\tau)$ обыкновенных дифференциальных уравнений в характеристической форме

$$
\begin{aligned}
& \left.\frac{d x_{1}}{\left(\partial H / \partial p_{1}\right)}=\frac{d x_{2}}{\left(\partial H / \partial p_{2}\right)}=\frac{d x_{3}}{\left(\partial H / \partial p_{3}\right)}=\frac{-d p_{1}}{\left(\partial H / \partial x_{1}\right.}\right) \\
& =\frac{-d p_{2}}{\left(\partial H / \partial x_{2}\right)}=\frac{-d p_{3}}{\left(\partial H / \partial x_{3}\right)}=\left[d \Phi / \sum_{q=1}^{3} p_{q}\left(\partial H / \partial p_{q}\right)\right] \\
& =d \tau .
\end{aligned}
$$

Система семи уравнений (11) соответствует уравнению в частных производных (8) в общем случае, когда показатель преломления $\bar{n}$ зависит от всех трех координат. Последнее возможно, если при отсутствии вращения среда неоднородна по $z$ и $\theta$.

Если в ИСО среда однородна и изотропна $(n=\mathrm{const})$, то в ВСО ее показатель преломления зависит лишь от координаты $x_{1} \equiv \rho \equiv \beta / K$, так что $\bar{n}^{2}=n^{2}+\bar{\alpha} \beta^{2}$ [12]. В такой среде вследствие $\left(\partial H / \partial x_{2}\right)=\left(\partial H / \partial x_{3}\right)=0$ и $d p_{2}=d p_{3}=0$ проекции $\nabla \boldsymbol{\Phi}$ на направления $\boldsymbol{\psi}_{0}$ и $\xi_{0}$ сохраняются как первые интегралы системы уравнений (11):

$$
\begin{gathered}
p_{2}=(\partial \Phi / \partial \psi) \equiv Q / K=\text { const }_{1} ; \\
p_{3}=(\partial \Phi / \partial \xi)-Z=\text { const }_{2} .
\end{gathered}
$$

В этом случае эйконал $\Phi \equiv \Phi(\beta, Q, Z)$, радиальная составляющая его градиента

$$
\begin{aligned}
& \frac{d \Phi}{d \rho} \equiv K \frac{d \Phi}{d \beta} \\
& = \pm \sqrt{-Z^{2}-\left(\frac{Q}{\beta}\right)^{2}+\frac{n^{2}-1+\bar{\alpha} \beta^{2}}{1-\beta^{2}}+(1+Q)^{2}} \\
& \equiv \pm|P(\beta, Q, Z)|
\end{aligned}
$$

и орт градиента эйконала

$$
\begin{aligned}
& \mathbf{n}_{0} \equiv \frac{\nabla \boldsymbol{\Phi}}{|\nabla \boldsymbol{\Phi}|}=\frac{P \boldsymbol{\rho}_{0}+(Q / \beta) \boldsymbol{\psi}_{0}+Z \boldsymbol{\zeta}_{0}}{\sqrt{(1+Q)^{2}+\left[\left(n^{2}-1+\bar{\alpha} \beta^{2}\right) /\left(1-\beta^{2}\right)\right]}} \\
& \equiv \mathbf{n}_{0}(\beta, Q, Z)
\end{aligned}
$$

зависят от одной координаты $\rho=\beta / K$ и двух параметров $(Z$ и $Q / K)$, а уравнения (11) трансформируются в систему пяти уравнений в характеристической форме

$$
\begin{aligned}
& \frac{d \beta}{2 K P}=\frac{d \xi}{2 Z}=\frac{d \psi}{2 K\left[\left(Q / \gamma^{2} \beta^{2}\right)-1\right]} \\
& =\frac{-d P}{-2 K\left\{\left(Q^{2} / \beta^{3}\right)+\beta\left[\left(n^{2}+\bar{\alpha}-1\right) /\left(1-\beta^{2}\right)^{2}\right]\right\}} \\
& =\frac{d \Phi}{2\left[P^{2}+Z^{2}-Q-Q^{2}+\left(Q^{2} / \beta^{2}\right)\right]}=d \tau=\frac{d s}{\Lambda(\beta, Q, Z)},
\end{aligned}
$$

в которой вторым независимым параметром может быть также длина дуги луча $s$.

\section{4. Траектория луча}

Координаты $\beta$, а также $\psi(\beta, Q, Z)$ и $\zeta(\beta, Q, Z)$, являющиеся решениями уравнений (15), однозначно связаны между собой как функции одного независимого параметра $\tau$ и при его изменении образуют в трехмерном пространстве кривую линию траектории луча (далее $R$-траекторию). В каждой точке $R$-траектории эйконал и составляющие его градиента, являясь функциями того же параметра $\tau$, имеют вполне определенные единственные значения, что позволяет найти другие свойства и характеристики решения уравнения (8).

Если в точке $\bar{A}$, имеющей координаты $\beta_{\bar{A}}=K \rho_{\bar{A}}$, $\psi_{\bar{A}}\left(\beta_{\bar{A}}\right) \quad$ и $\xi_{\bar{A}}\left(\beta_{\bar{A}}\right)$, проекции градиента эйконала $\nabla \boldsymbol{\Phi}_{\bar{A}}=P_{\bar{A}} \boldsymbol{\rho}_{0}+\left(Q_{\bar{A}} / \beta_{\bar{A}}\right) \boldsymbol{\psi}_{0}+Z_{\bar{A}} \boldsymbol{\zeta}_{0} \quad$ удовлетворяют уравнению (9), то зависимости $\psi_{\bar{A}}(\beta)$ и $\xi_{\bar{A}}(\beta)$ являются параметрическим представлением в пространстве переменных $\beta, \psi, \xi R$-траектории, проходящей через точку $\bar{A}$. Среди неограниченного числа таких $R$-траекторий существует одна особая, которая из точки $\bar{A}$ приходит в точку $\bar{B}$, имеющую координаты $\beta_{\bar{B}}, \psi_{\bar{B}}, \xi_{\bar{B}}$. Пространственные координаты этой $R$-траектории описываются решениями $\psi_{\overline{A B}}(\beta)$ и $\xi_{\overline{A B}}(\beta)$ уравнений $(15)$, которые содержат вполне конкретные (априори неизвестные) постоянные параметры $Z_{\overline{A B}}(\beta)$ и $Q_{\overline{A B}}$. Для их определения можно использовать независимость первых интегралов (12) от скорости вращения системы координат и однозначно определить $Z_{A B}$ и $Q_{A B}$ в ИСО, где $K=0$ и орт градиента эйконала (8.2) в координатах $\rho, \theta, z$ совпадает с ортом вектора $\mathbf{r}_{B}-\mathbf{r}_{A}$ :

$$
\begin{aligned}
& \frac{1}{n}\left\{\frac{\partial \Phi_{0}}{\partial \rho} \boldsymbol{\rho}_{0}+\frac{1}{\rho} \frac{\partial \Phi_{0}}{\partial \theta} \boldsymbol{\theta}_{0}+\frac{\partial \Phi_{0}}{\partial z} \mathbf{z}_{0}\right\} \\
& =\frac{\left(\boldsymbol{\rho}_{B}-\boldsymbol{\rho}_{A}\right)+\left(z_{B}-z_{A}\right) \mathbf{z}_{0}}{\sqrt{\left(z_{B}-z_{A}\right)^{2}+\left|\boldsymbol{\rho}_{B}-\boldsymbol{\rho}_{A}\right|^{2}}} .
\end{aligned}
$$


Проекции (16) на направления $\mathbf{z}_{0}$ и $\boldsymbol{\theta}_{0}\left(\boldsymbol{\psi}_{0}\right.$ и $\boldsymbol{\zeta}_{0}$ в ВСО) дают два уравнения

$$
\begin{aligned}
& \frac{\partial \Phi_{0}}{\partial z}=\frac{\partial \Phi}{\partial \xi} \equiv Z_{A B} \\
& =\frac{n\left(z_{B}-z_{A}\right)}{\sqrt{\left(z_{B}-z_{A}\right)^{2}+\rho_{B}^{2}+\rho_{A}^{2}-2 \rho_{A} \rho_{B} \cos \left(\theta_{B}-\theta_{A}\right)}}, \\
& \frac{\partial \Phi}{\partial \theta}=\frac{\partial \Phi}{\partial \psi} \equiv \frac{Q_{A B}}{K} \\
& =\frac{n \rho\left[\left(\boldsymbol{\theta}_{0} \bullet \boldsymbol{\rho}_{B}\right)-\left(\boldsymbol{\theta}_{0} \bullet \boldsymbol{\rho}_{A}\right)\right]}{\sqrt{\left(z_{B}-z_{A}\right)^{2}+\rho_{B}^{2}+\rho_{A}^{2}-2 \rho_{A} \rho_{B} \cos \left(\theta_{B}-\theta_{A}\right)}}
\end{aligned}
$$

для определения постоянных $Z_{A B}$ и $Q_{A B} / K$ через полярные координаты $\rho_{A}$ и $\theta_{A, B}$ векторов $\boldsymbol{\rho}_{\boldsymbol{A}, \boldsymbol{B}}$ в ИСО в плоскости, ортогональной направлению $\mathbf{z}_{0}$. Если в треугольнике $A O B$, образованном векторами $\boldsymbol{\rho}_{A}, \boldsymbol{\rho}_{B}$ и $\boldsymbol{\rho}_{A B}=\boldsymbol{\rho}_{B}-\boldsymbol{\rho}_{A}$, ввести углы $\alpha_{A, B}$ при вершинах $A$, $B$ и установить угол $\theta=0$ по направлению высоты $h=\rho_{A} \sin \alpha_{A}=\rho_{B} \sin \alpha_{B}$, опущенной из вершины $O$ на сторону $A B$, то координаты $\theta_{A, B}$ можно определить в виде $\theta_{A, B}= \pm\left[\alpha_{A, B}-(\pi / 2)\right]$ и далее с учетом $\cos \left(\theta_{B}-\theta_{A}\right)=-\cos \left(\alpha_{B}+\alpha_{A}\right)$ преобразовать (17) в

$$
\begin{aligned}
& \frac{Q_{A B}}{K}=\frac{n h\left[\rho_{B} \cos \alpha_{B}+\rho_{A} \cos \alpha_{A}\right]}{\sqrt{\left(z_{B}-z_{A}\right)^{2}+\rho_{B}^{2}+\rho_{A}^{2}+2 \rho_{A} \rho_{B} \cos \left(\alpha_{B}+\alpha_{A}\right)}} \\
& \equiv \frac{Z_{A B}}{Z_{B}-Z_{A}} h\left[\rho_{B} \cos \alpha_{B}+\rho_{A} \cos \alpha_{A}\right] .
\end{aligned}
$$

Из (17) и (18) следует, что $Z_{A B}=0$ и $\left(Q_{A B} / K\right)=n h$ в случае $z_{B}=z_{A}$, когда $R$-траектория располагается в плоскости, перпендикулярной оси $O Z$. В другом случае $\left(\boldsymbol{\rho}_{B} / \rho_{B}\right)=\left(\boldsymbol{\rho}_{A} / \rho_{A}\right)$, когда $R$-траектория находится в плоскости $\theta=$ const $_{\theta}\left(\psi=\right.$ const $_{\psi}$ в $\left.\mathrm{BCO}\right)$, из (17) и (18) получится $Q_{A B}=0$.

Если в некоторый момент времени $t_{0}$ точки $\bar{A}, \bar{B}$ и начало отсчета координаты $\psi$ в ВСО (с точностью до возможной малой неоднородности пространства из-за вращения) совпадают с точками $A, B$ и $\theta=0$ в ИСО, то в формулах (17) будут справедливы замены $A, B$, $\boldsymbol{\theta}_{0} \leftrightarrow \bar{A}, \bar{B}, \boldsymbol{\psi}_{0}$ и соответственно верны равенства

$$
\begin{aligned}
& Z_{\overline{A B}} \cong Z_{A B} ; Q_{\overline{A B}} \cong Q_{A B} ;\left|P\left(\beta, Q_{\overline{A B}}, Z_{\overline{A B}}\right)\right| \\
& \cong\left|P\left(\beta, Q_{A B}, Z_{A B}\right)\right| .
\end{aligned}
$$

В ЦСК $R$-траектория $\bar{A} \bar{B}$ имеет несколько параметров своей структуры, из которых большое значение в расчетах имеет минимальное расстояние до оси $O Z$

$$
\sqrt{\beta_{\bar{A} \bar{B}} \cong \beta_{A B}=} \sqrt{\frac{-\sqrt{\left(n^{2}-Z_{A B}^{2}\right)^{2} / 4+Q_{A B}^{2}\left(n^{2}+\bar{\alpha}\right)+Q_{A B}\left(n^{2}-Z_{A B}^{2}\right)}}{\left(1+Q_{A B}\right)^{2}-\bar{\alpha}-Z_{A B}^{2}}} .
$$

$\mathrm{B}$ области $\beta_{\bar{A}} \geq \beta \geq \beta_{\overline{A B}}$ радиальная проекция луча $\overline{A B}$ направлена к оси вращения $\left(\boldsymbol{\rho}_{0} P \overline{A B}=-\rho_{0}\left|P_{\overline{A B}}\right|\right)$, а в области $\beta_{\overline{A B}} \leq \beta \leq \beta_{\bar{B}}$ поток энергии $\boldsymbol{\rho}_{0} P_{\overline{A B}}=\boldsymbol{\rho}_{0}\left|P_{\overline{A B}}\right|$ имеет противоположное направление. Поэтому для расчета двух частей $R$-траектории следует использовать две пары интегралов уравнений (15), имеющих переменный верхний предел $\beta$ и разные нижние пределы $\beta_{\bar{A}}, \beta_{\bar{B}}$. Их можно представить в единой форме

$$
\begin{gathered}
\xi_{\overline{A B}}(\beta) \cong \int_{\beta_{\bar{S}}}^{\beta} \frac{Z_{A B}}{\left|P_{A B}\left(\beta^{\prime}\right)\right|} d \beta^{\prime} ; \\
\psi_{\overline{A B}}(\beta) \cong-\int_{\beta_{\bar{S}}}^{\beta} \frac{1}{\left|P_{A B}\left(\beta^{\prime}\right)\right|}\left\{\frac{Q_{A B}}{\left(\beta^{\prime}\right)^{2}}-\left(1+Q_{A B}\right)\right\} d \beta^{\prime},
\end{gathered}
$$

где $\quad \beta_{\bar{S}} \equiv \beta_{\bar{A}} \quad$ Для $\quad \beta_{\bar{A}} \geq \beta \geq \beta_{\overline{A B}} \quad$ и $\quad \beta_{\bar{S}} \equiv \beta_{\bar{B}} \quad$ для $\beta_{\overline{A B}} \leq \beta \leq \beta_{\bar{B}}$. Заметим, что аналитические решения (21) в виде линейных комбинаций эллиптических интегралов не вносят дополнительной ясности в схему расчета $R$-траектории и здесь не приводятся.

При описании „встречного“ луча (из пункта $B$ в пункт $A$ ) в уравнениях (17) следует переставить индексы $(A \leftrightarrow B)$ и для расчета этой $R$-траектории использовать параметры $Z_{B A}=-Z_{A B}$ и $Q_{B A}=-Q_{A B}$. Тогда в случае $Q_{A B} \geq 0$ в соответствии с (13), (20) получится $\left|P_{A B}(\beta)\right| \geq\left|P_{B A}(\beta)\right|$ и $\beta_{A B}^{2} \leq \beta_{B A}^{2}$, так что, согласно (21), „встречны““ $R$-траектории в ВСО между пунктами $\bar{A}$ и $\bar{B}$ будут различны при всех $\beta$ в интервале, ограниченном значениями $\beta_{\bar{A}} \cong \beta_{A}, \beta_{\bar{B}} \cong \beta_{B}$.

Рассмотрим результаты расчетов по формулам (21) „встречных“ $R$-траекторий между точками $\bar{A}$ и $\bar{B}$ в плоскости $\xi=$ const внутри диэлектрика $\left(n^{2}=3, \bar{\alpha}=0.01\right)$, вращающегося с угловой скоростью $\Omega=3 \cdot 10^{8} \mathrm{c}^{-1}$ $\left(K=10^{-2} \mathrm{~cm}^{-1}\right)$. Векторы $\boldsymbol{\rho}_{A}$ и $\boldsymbol{\rho}_{B}$, определяющие координаты точек $A$ и $B$ в ИСО, выберем отличающимися в 2 раза по абсолютной величине $\left(\left|\rho_{B}\right|=2\left|\rho_{A}\right|=4 \mathrm{~cm}\right)$ и ортогональными друг другу $\left(\boldsymbol{\rho}_{A} \cdot \boldsymbol{\rho}_{B}\right)=0$. В этом случае расчетными параметрами „встречных“ $R$-траекторий будут $Z_{A B}=0$ и $Q_{A B}=-Q_{B A}=K(48 / 5)^{1 / 2}>0$, так что форму $R$-траектории каждого „встречного“ луча на плоскости $\beta, \psi$ определяет соответствующая зависимость $\psi_{\overline{A B}}(\beta)$ или $\psi_{\overline{B A}}(\beta)$.

Зависимость $\psi_{\overline{A B}}(\beta), \quad$ начинающаяся в точке $\bar{A} \cong A\left(\beta_{A}=0.02 ; \psi_{A}=-\operatorname{arctg} 0.5\right), \quad$ на другой границе $\beta_{B}=0.04$ интервала изменения $\beta$ заканчивается в точке $\bar{B}_{R}$, азимутальная координата которой $\psi_{\overline{A B}}\left(\beta_{B}\right) \quad$ больше координаты $\psi_{B}=\operatorname{arctg} 2$ точки $\bar{B} \cong B \quad$ на $\quad \delta \psi_{\overline{A B}} \cong 3.12 \cdot 10^{-4}$. Следовательно, ВСО область определения $R$-траектории $\overline{A B}$ по координате $\psi$ увеличивается по сравнению с таким же параметром $R$-траектории $A B$ в ИСО. Наличие относительно большого (на 5 порядков превышающего точность вычислений) $\delta \psi_{\overline{A B}}$ может оказаться принципиально важным, например, при создании в ВСО ретрансляционной сети, поскольку именно в пункт 
$\bar{B}_{R}$ прибудет сигнал из $\bar{A}$. „Встречная“ зависимость $\psi_{\overline{B A}}(\beta), \quad$ начинаясь $\quad$ в пункте $\quad \bar{B} \cong B\left(\beta_{B}=0.04\right.$; $\left.\psi_{B}=(\pi / 2)-\operatorname{atrctg} 2\right), \quad$ заканчивается при $\beta_{A}=0.02$ в пункте $\bar{A}_{R}$, азимутальная координата которого больше $\psi_{A}$ на $\delta \psi_{\overline{B A}} \cong 1.28 \cdot 10^{-3}$. Таким образом, области определения по координате $\psi$ „встречных“ $R$-траекторий $\quad \Delta \psi_{\overline{A B}} \quad$ и $\quad \Delta \psi_{\overline{B A}}=-(\pi / 2)+\delta \psi_{\overline{B A}}$, являющиеся структурными параметрами обеих зависимостей $\psi_{\overline{B A}}(\beta)$ и $\psi_{\overline{B A}}(\beta)$, заметно отличаются от разности координат $\psi_{B}-\psi_{A}=(\pi / 2)$ пунктов $\bar{A}$ и $\bar{B}$.

Рассматриваемые $R$-траектории зависят (в том числе через параметр $Q_{A B}$ ) от показателя преломления диэлектрика $n$. Для установления вклада $n$ в их характеристики рассмотрим $R$-траектории в вакууме, где $\hat{n}^{2}=1, \hat{\bar{\alpha}}=0$, $\left(\hat{Q}_{\overline{A B}}\right) \approx\left(\hat{Q}_{A B}\right)=K h=K \sqrt{16 / 5}$ и $\hat{Z}_{\overline{A B}}=0$. В этом случае в каждой области $\beta_{\bar{S}}^{2} \geq \beta^{2} \geq \hat{\bar{\beta}}_{1,2}^{2} \equiv \hat{Q}_{1,2}^{2} /\left(1+\hat{Q}_{1,2}\right)^{2}$, где „встречные“ $R$-траектории определены, зависимости $\hat{\psi}_{1,2}(\beta)$ в случае $\hat{Q}_{1}=-\hat{Q}_{2} \geq 0$ находятся с помощью аналитических решений (21) таких, как

$$
\hat{\psi}_{1,2}(\beta)=\sqrt{\beta^{2}-\hat{\bar{\beta}}_{1,2}^{2}} \mp \operatorname{arctg} \sqrt{\left(\beta / \hat{\bar{\beta}}_{1,2}\right)^{2}-1}+\hat{C}_{1,2} .
$$

Области определения по $\psi$ „встречных“ вакуумных $R$-траекторий $\Delta \hat{\psi}_{\overline{A B}}$ и $\Delta \hat{\psi}_{\overline{B A}}$, вычисленные по формулам (22), отличаются от $( \pm \pi / 2)$ на положительные величины $\delta \hat{\psi}_{\overline{A B}} \cong 2.712 \cdot 10^{-3}$ и $\delta \hat{\psi}_{\overline{B A}} \cong 3.128 \cdot 10^{-3}$, которые в несколько раз превышают значения $\delta \psi_{\overline{A B}}$ и $\delta \psi_{\overline{B A}}$ „встречных“ $R$-траекторий в диэлектрике.

На рис. 1 в полярных координатах $\rho, \psi$ изображены „встречные“ $R$-траектории для диэлектрика (сплошные линии 1,2 ) и вакуума (пунктирные линии 3,4 ). Траектории из $\bar{A}$ в $\bar{B}$ (линии 1,3 ) смещены относительно прямой $\overline{A B}$ по направлению вращения $\boldsymbol{\psi}_{0}$ и не имеют общих точек со „Встречными“ $R$-траекториями (линии 2,4 ), смещенными от $\overline{A B}$ в противоположную сторону.

Единственной причиной, определяющей в вакууме структурные параметры $R$-траекторий $\hat{\psi}_{3,4}(\beta)$, является неоднородность пространства неинерциальной ВСО. Она проявляется в виде анизотропии для лучей (и волн), у которых орты их лучевых векторов $\mathbf{s}_{0}($ и $\nabla \boldsymbol{\Phi})$ имеют проекции на азимутальное направление $\boldsymbol{\psi}_{0}$. Из сопоставления „встречных“ вакуумных $R$-траекторий $\hat{\psi}_{\overline{A B}}(\beta)$ и $\hat{\psi}_{\overline{B A}}(\beta)$ друг с другом и с соответствующими $R$-траекториями $\psi_{\overline{A B}}(\beta)$ и $\psi_{\overline{B A}}(\beta)$ в диэлектрике виден не только общий результат воздействия такой анизотропии на распространение лучей, но и проявляется второй физический механизм ее образования в ВСО, который известен (после опытов Физо) как увлечение света движущейся средой. Очевидно, что движение диэлектрического вещества дает вклад (по направлению $\boldsymbol{\psi}_{0}$ ) в азимутальные проекции обоих лучей (и в $\nabla \boldsymbol{\Phi})$, изменяя их локальные направления $\mathbf{s}_{0}\left(\beta, \pm Q_{A B}, \pm Z_{A B}\right)$ и $\mathbf{n}_{0}\left(\beta, \pm Q_{A B}, \pm Z_{A B}\right)$ относительно „опорных“ направлений $\pm \mathbf{s}_{0}^{0} \equiv \pm \mathbf{n}_{0}^{0}$ в ИСО. Увлечение света движущейся средой выравнивает области определения по $\psi$ „встреч-

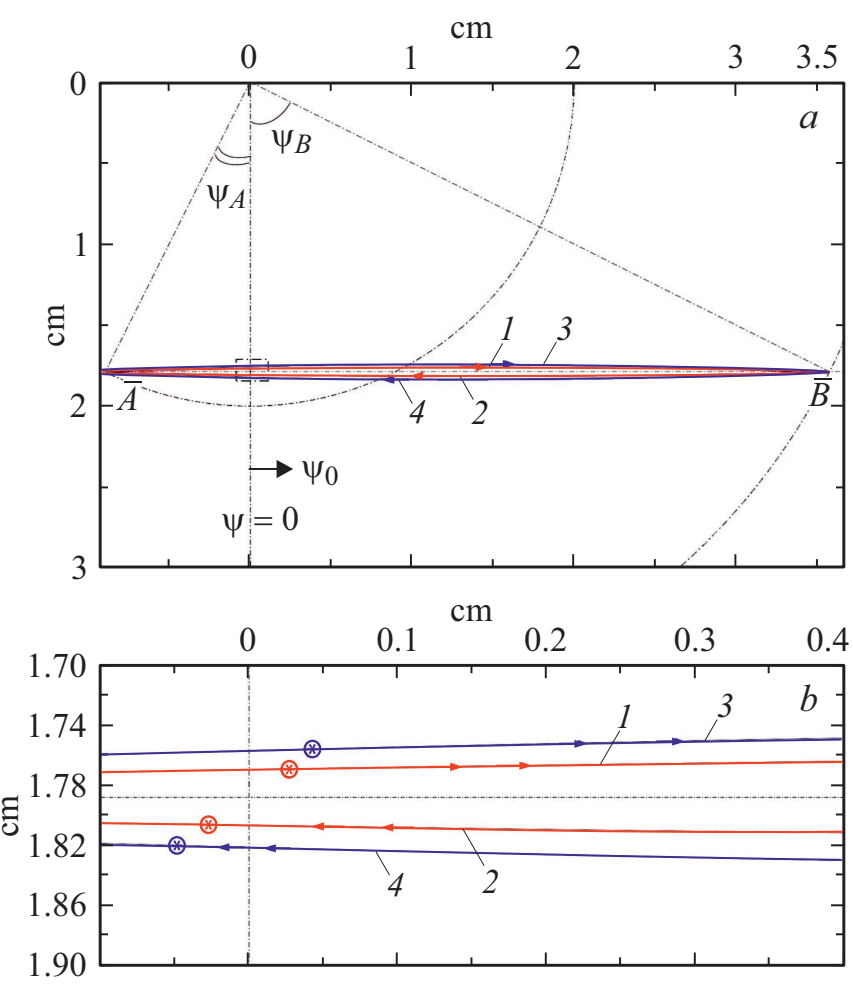

Рис. 1. „Встречные“ $R$-траектории лучей в диэлектрике (сплошные линии 1,2 ) и в вакууме (пунктирные линии 3,4 ) в полярных координатах $(\rho, \psi)$ вращающейся системы отсчета в пространстве между пунктами $\bar{A}$ и $\bar{B}(a)$; в увеличенном масштабе в границах выделенного фрагмента с указанием точек траекторий, находящихся на минимальном расстоянии от оси вращения $(b)$.

ных“ $R$-траекторий, уменьшая анизотропию в ВСО, обусловленную неоднородностью свободного пространства.

\section{5. Длина дуги траектории луча}

Важной характеристикой луча является орт $\mathbf{s}_{0}$ лучевого вектора, который указывает направление потока мощности (вектора Пойнтинга). Орт $\mathbf{s}_{0}$ определяется как предел приращения $d \mathbf{r}$ вектора координат движущейся световой точки $\mathbf{r}(s)$ к длине дуги траектории луча $d s \equiv|d \mathbf{r}|$, что позволяет найти его в виде

$$
\begin{gathered}
\mathbf{s}_{0}=\frac{K d \mathbf{r}}{K d s}=\frac{K\left(\boldsymbol{\rho}_{0} d \rho+\boldsymbol{\psi}_{0} \rho d \psi+\xi_{0} d \xi\right)}{\sqrt{(d \beta)^{2}+(\beta d \psi)^{2}+(K d \xi)^{2}}} \\
\equiv \frac{K\left(\boldsymbol{\rho}_{0} d \rho+\boldsymbol{\psi}_{0} \rho d \psi+\boldsymbol{\xi}_{0} d \xi\right)}{d \beta \sqrt{1+\beta^{2}(d \psi / d \beta)^{2}+K^{2}(d \xi / d \beta)^{2}}} .
\end{gathered}
$$

Из (23), (15) и (9) с учетом (19) находятся уравнения для элемента длины дуги луча

$$
\begin{aligned}
& K \frac{d s_{\overline{A B}}}{d \beta} \cong \frac{\mp 1}{\left|P_{A B}\left(Q_{A B}, Z_{A B}, \beta\right)\right|} \\
& \times \sqrt{\begin{array}{c}
1-Q_{A B}^{2}+\beta^{2}\left(1+Q_{A B}\right)^{2} \\
+\left[\left(n^{2}-1+\bar{\alpha} \beta^{2}\right) /\left(1-\beta^{2}\right)\right]
\end{array}},
\end{aligned}
$$


где фактор $(-1)$ относится к первой части траектории $\overline{A B}$ (области $\left.\beta_{\bar{A}} \geq \beta \geq \beta_{\overline{A B}}\right)$, a $(+1) \quad-$ ко второй. Длины дуг $S_{\overline{A B}}$ и $S_{\overline{B A}}$ „встречных“ $R$-траекторий вычисляются по правилам, подобным (21). В диэлектрике длина $S_{\overline{A B}} \cong 4.472 \mathrm{~cm}>S_{\overline{B A}}$ на $1.8 \cdot 10^{-3} \mathrm{~cm}$, в вакууме длины дуг „встречных“ $R$-траекторий уменьшаются $\left(\hat{S}_{\overline{A B}}<S_{\overline{A B}}\right)$, а их различие $\hat{S}_{\overline{A B}}-\hat{S}_{\overline{B A}} \cong 2.7 \cdot 10^{-3} \mathrm{~cm}$ увеличивается. В ИСО дуги „Встречных“ $R$-траекторий равны: $\left(S_{1,2}\right)_{K=0}=5.577 \mathrm{~cm}$ для $n^{2}=3$ и $\left(\hat{S}_{1,2}\right)_{K=0}=\sqrt{20} \mathrm{~cm}$ в вакууме.

Таким образом, в ВСО из-за неоднородности пространства длины дуг „встречных“ $R$-траекторий уменьшаются по сравнению с их размерами в ИСО. Причем у луча, имеющего поток мощности по направлению вращения, длина дуги несколько больше длины дуги „встречного“ луча, и эта разница максимальна в вакууме. Увлечение света движущейся средой эффективно выравнивает длины дуг „встречных“ $R$-траекторий.

Для объяснения приведенных выше результатов следует иметь в виду, что $\hat{\psi}_{\overline{A B}}(\beta)$ и $\hat{\psi}_{\overline{B A}}(\beta)$ являются двумя представлениями в полярных координатах $\rho, \psi$ мгновенного снимка проекций двух мировых линий светового импульса в свободном 4-пространстве ВСО $(\rho, \psi, \xi, i c t)$ на плоскость, ортогональную оси вращения. Каждая зависимость $\hat{\psi}_{1,2}(\beta)$ может иметь смысл следа лучевой трубки, которую импульс „прожигает“ в вакууме (во вращающейся среде), или лучевой трубки, заполненной движущимися импульсами. Световой импульс после старта в пункте $\bar{A}$ в момент $t_{\bar{A}}=0$ через некоторое время $\hat{T}_{\overline{A \Gamma}}$ оказывается в поперечном сечении $\bar{\Gamma}$ лучевой трубки $\overline{A B}$, имеющем координаты $\beta_{\bar{\Gamma}}, \hat{\psi}_{\overline{A B}}\left(\beta_{\bar{\Gamma}}\right), \hat{\xi}_{\overline{A B}}\left(\beta_{\bar{\Gamma}}\right)$. Время $\hat{T}_{\overline{A \Gamma}}$ можно определить, используя условие равенства нулю квадрата интервала между двумя событиями на мировой линии импульса. В 4-пространстве ВСО, имеющем метрический тензор $g_{i k}\left(g_{00}=-1+K^{2} \rho^{2}, g_{02}=g_{20}=K \rho^{2}\right.$, $\left.g_{22}=\rho^{2}, g_{11}=g_{33}=1\right)$, это условие можно представить в виде уравнения связи дифференциальных приращений трех координат и времени на $R$-траектории $\overline{A \bar{B}}$

$$
\begin{aligned}
& \left(1-K^{2} \rho^{2}\right)\left(c d \hat{t}_{\overline{A B}}\right)^{2}-2 K \rho^{2}\left(d \hat{\psi}_{\overline{A B}}\right)\left(c d \hat{t}_{\overline{A B}}\right)-(d \rho)^{2} \\
& -\rho^{2}\left(d \hat{\psi}_{\overline{A B}}\right)^{2}-\left(d \hat{\zeta}_{\overline{A B}}\right)^{2}=0 .
\end{aligned}
$$

Из (25) с учетом (15) находятся уравнения для элементарного временного интервала

$$
\left(d \hat{t}_{\overline{A B}} / d \beta^{2}\right)=\mp(1 / 2 c K)\left(1 /{\sqrt{\beta^{2}-\hat{\beta}_{\overline{A B}}}}^{2}\right),
$$

необходимого импульсу для прохождения элемента $d \hat{s}_{\overline{A B}}$ на первом (-) или втором $(+)$ участках $R$-траектории $\overline{A B}$. Решения (26) позволяют найти время

$$
\hat{T}_{\overline{A \Gamma}}=(1 / c K)\left(\sqrt{\beta_{\bar{A}}^{2}-\hat{\beta}_{\overline{A B}}^{2}} \mp \sqrt{\beta_{\bar{\Gamma}}^{2}-\hat{\beta}_{\overline{A B}}^{2}}\right),
$$

за которое световой импульс приходит из пункта $\bar{A}$ в пункт $\bar{\Gamma}$, находящийся на первой (или второй) части $R$-траектории $\overline{A B}$.
Если положение светового импульса на $R$-траектории $\overline{A B}$ в точке $\bar{\Gamma}$ спроецировать в точку $\Gamma$ на ортогональной оси вращения плоскости $(\beta, \theta)$ в ИСО, то эта проекция с учетом поворота осей ВСО будет иметь координаты $\hat{\theta}\left(\beta_{\Gamma}\right)=\hat{\psi}_{\overline{A B}}\left(\beta_{\bar{\Gamma}}\right)+\Omega \hat{T}_{\overline{A \Gamma}}$ и $\beta_{\Gamma}=\beta_{\bar{\Gamma}}$. Такая регистрация координат движущегося импульса позволяет представить „встречные“ $R$-траектории $A B$ и $B A$ в ИСО как функции координат $\beta, \theta$.

Из (25) и (15) получаются уравнения для элементарного временного интервала

$$
\mp c K \frac{d t_{\overline{A B}}}{d \beta} \cong \frac{Q_{A B}-\beta^{2}\left(1+Q_{A B}\right)+\sqrt{n^{2}+\bar{\alpha} \beta^{2}}}{\left|P_{A B}\left(Q_{A B}, Z_{A B}, \beta\right)\right|\left(1-\beta^{2}\right)},
$$

который требуется световому импульсу для прохождения отрезка $d s_{\overline{A B}}$ длины дуги $R$-траектории $\overline{A B}$ во вращающемся диэлектрике. Уравнения (28) аналогичны по содержанию уравнениям (24) в том смысле, что обеспечивают однозначное соответствие длины дуги $S_{\overline{A \Gamma}}$ между точками $\bar{A}$ и $\bar{\Gamma}$ на $R$-траектории $\overline{A B}$ и временем $T_{\overline{A \Gamma}}$, за которое световой импульс преодолеет длину $S_{\overline{A \Gamma}}$. С помощью (28) можно вычислить времена $T_{\overline{A B}}$ и $T_{\overline{B A}}$, за которые импульс проходит длины дуг $S_{\overline{A B}}$ и $S_{\overline{B A}}$ „встречных“ $R$-траекторий в диэлектрике.

\section{6. Изменение интенсивности на траектории луча}

Под интенсивностью света $\bar{I}$ в ВСО понимается поток мощности излучения [17] в виде среднего за период оптического колебания вектора Пойнтинга $\overline{\bar{S}}^{2 \pi / \omega} \equiv \bar{I} \cdot \mathbf{s}_{0}$. В стационарной консервативной среде $\bar{I} \mathbf{s}_{0}$ сохраняется, что эквивалентно уравнению

$$
\begin{aligned}
& \operatorname{div}\left(\bar{I} \cdot \mathbf{s}_{0}\right) \equiv\left(\nabla \bar{I} \cdot \mathbf{s}_{0}\right)+\bar{I} \operatorname{div} \mathbf{s}_{0} \\
& \equiv \frac{d \bar{I}}{d s}+\frac{\bar{I}}{\rho}\left\{\frac{\partial\left[\rho\left(\boldsymbol{\rho}_{0} \cdot \mathbf{s}_{0}\right)\right]}{\partial \rho}+\frac{\partial\left(\boldsymbol{\psi}_{0} \cdot \mathbf{s}_{0}\right)}{\partial \psi}+\frac{\partial\left[\rho\left(\xi_{0} \cdot \mathbf{s}_{0}\right)\right]}{\partial \xi}\right\} \\
& =0 .
\end{aligned}
$$

Компоненты $\mathbf{s}_{0}$, согласно $(23)$ и $(15)$, не зависят от $\psi$ и $\xi$, а $\left[\rho\left(\boldsymbol{\rho}_{0} \bullet \mathbf{s}_{0}\right)\right]$ преобразуется в

$$
\begin{aligned}
& \frac{\rho P_{\overline{A B}}}{\sqrt{P_{\overline{A B}}^{2}+\left[\left(Q_{\overline{A B}} / \beta\right)-\beta\left(1+Q_{\overline{A B}}\right)\right]^{2}+Z_{\overline{A B}}^{2}}} \\
& \equiv\left(\rho \frac{d \rho}{d s}\right) \equiv \frac{1}{2}\left(\frac{d \rho^{2}}{d s}\right) .
\end{aligned}
$$

Уравнение (29) с учетом (30) трансформируется в уравнение

$$
\begin{aligned}
& \frac{\operatorname{div}\left(\bar{I} \cdot \mathbf{s}_{0}\right)}{\bar{I}}=\frac{d(\ln \bar{I})}{d p^{2}} \cdot\left(\frac{d \rho^{2}}{d s}\right)+\frac{d}{d \rho^{2}}\left(\frac{d \rho^{2}}{d s}\right) \\
& \equiv\left(\frac{d \rho^{2}}{d s}\right)\left\{\frac{d}{d \rho^{2}} \ln \left[\bar{I}\left(\frac{d \rho^{2}}{d s}\right)\right]\right\}=0,
\end{aligned}
$$


имеющее при $\left(d \rho^{2} / d s\right) \neq 0$ решение

$$
\begin{aligned}
& \bar{I}\left(\rho^{2}\right)=\bar{C}_{\overline{A B}}\left(d s / d \rho^{2}\right) \equiv\left(\bar{C}_{\overline{A B}} K / 2 \beta P_{\overline{A B}}\right) \\
& \times \sqrt{P_{\overline{A B}}^{2}+\left[\left(Q_{\overline{A B}} / \beta\right)-\beta\left(1+Q_{\overline{A B}}\right)\right]^{2}+Z_{\overline{A B}}^{2}},
\end{aligned}
$$

в котором постоянная $\bar{C} \overline{\overline{A B}}$ определяется через интенсивность $\bar{I}\left(\rho \frac{2}{A}\right)$ в точке $\bar{A}$.

\section{7. Траектория движения волнового фронта}

В отличие от траекторий лучей, по которым распространяются экспериментально наблюдаемые потоки мощности электромагнитных волн, в анизотропной среде существуют не совпадающие с ними (в общем случае) фазовые $f$-траектории перемещения волновых фронтов. В каждой точке $f$-траектории направление перемещения волнового фронта указывает орт $\mathbf{n}_{0}(\beta, Q, Z)$ градиента эйконала (14), имеющий смысл орта по направлению касательной к этой виртуальной линии. Можно считать, что $f$-траекторию в пространстве вращающихся цилиндрических координат описывает основание вектора $\mathbf{n}_{0}$ подобно тому, как основание орта лучевого вектора $\mathbf{s}_{0}$ описывает $R$-траекторию.

Для определения $f$-траектории $A \bar{B}$ можно воспользоваться лучевой $R$-траекторией $\overline{A B}$ и в каждой ее точке, имеющей координаты $\beta, \psi_{\overline{A B}}(\beta), \xi_{\overline{A B}}(\beta)$, учесть разницу в направлениях ортов $\mathbf{s}_{0}$ и $\mathbf{n}_{0}$. Проекции $\mathbf{s}_{0 \perp}$ и $\mathbf{n}_{0 \perp}$ ортов, ортогональные оси вращения, образуют угол $v_{\overline{A B}}(\beta)$, абсолютную величину и знак которого можно определить с помощью скалярного $\left(\mathbf{s}_{0 \perp} \bullet \mathbf{n}_{0 \perp}\right)$ и векторного $\left[\mathbf{s}_{0 \perp} \times \mathbf{n}_{0 \perp}\right]$ произведений, например, в виде

$$
\begin{aligned}
\nu_{\overline{A B}}(\beta)=\operatorname{arctg} & \frac{\mp\left|P_{\overline{A B}}(\beta)\right|\left(1+Q_{\overline{A B}}\right)}{-Z_{\overline{A B}}^{2}+1+Q_{\overline{A B}}} . \\
+\left[\left(n^{2}-1+\bar{\alpha} \beta^{2}\right) /\left(1-\beta^{2}\right)\right] & .
\end{aligned}
$$

B (33) знак $(-1)$ относится к первой части R-траектории (области $\beta_{\bar{A}} \geq \beta \geq \beta_{\overline{A B}}$, а знак $(+1)-$ ко второй. На угол $\nu_{\overline{A B}}(\beta)$ отличаются азимутальные углы

$$
v_{\overline{A B}}(\beta) \equiv \psi_{\overline{A B}}+v_{\overline{A B}}(\beta)
$$

сопряженных точек $f$ - и $R$-траекторий $\overline{A B}$. Две другие координаты $\beta$ и $\xi_{\overline{A B}}(\beta)$ сопряженных точек одинаковы, поскольку обе являются решениями (15) и (21).

На рис. 2 изображены в полярных координатах $(\rho, \psi)$ „встречные $f$-траектории $\overline{A B}$ (пунктирные линии 5,6 ) и ,встречные ${ }^{\text {“ }} R$-траектории $\overline{A B}$ (сплошные линии 3,4$)$ в вакууме. Видно, что $f$-траектории $(5,6)$ и $R$-траектории $(3,4)$ не имеют общих точек. В пунктах $\hat{\beta}_{\overline{A B}}$ и $\hat{\beta}_{\overline{B A}}$, где $\left|P_{\overline{A B}}\left(\hat{\beta}_{\overline{A B}}\right)\right|=0=\left|P_{\overline{B A}}\left(\hat{\beta}_{\overline{B A}}\right)\right|$, сплошные и пунктирные траектории одной направленности по отношению к вектору $\left(\boldsymbol{\rho}_{B}-\boldsymbol{\rho}_{A}\right)$ касаются друг друга.

Вывод уравнения для изменения $d \sigma$ длины дуги $f$-траектории $\overline{A B}$ следует начать с введения орта по направлению касательной к длине дуги этой виртуальной

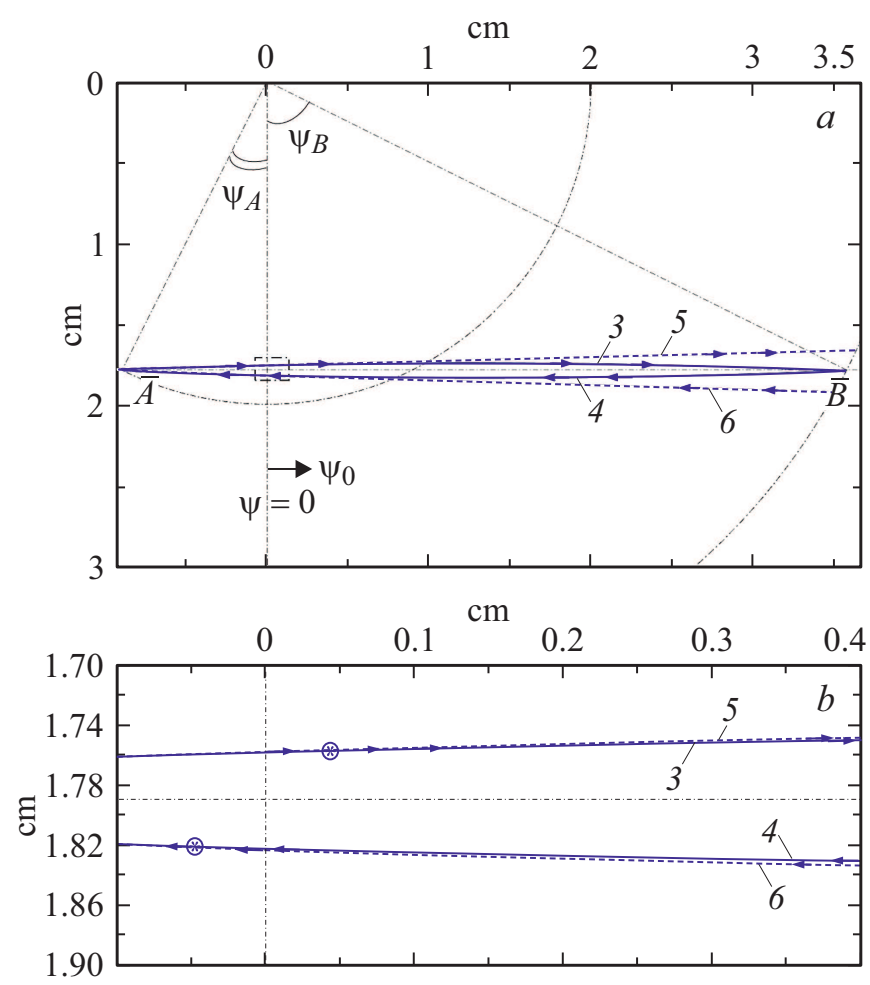

Рис. 2. „Встречные“ $R$-траектории лучей (сплошные линии 3,4$)$ и $f$-траектории фазовых фронтов (пунктирные линии 5,6$)$ в полярных координатах $(\rho, \psi)$ вращающейся системы отсчета в пространстве между пунктами $\bar{A}$ и $\bar{B}(a)$; в увеличенном масштабе в границах выделенного фрагмента с указанием точек касания попутных траекторий, находящихся на минимальном расстоянии от оси вращения $(b)$.

линии. По аналогии с (23) орт можно определить как предел приращения $d \mathbf{r}$ вектора координат движущейся точки $f$-траектории $\mathbf{r}(\sigma)$ к элементу $d \sigma$ длины ее дуги и далее приравнять его вектору $\mathbf{n}_{0}$ :

$$
\begin{aligned}
& \frac{d \mathbf{r}}{d \sigma}=\frac{K d \mathbf{r}}{K \sqrt{|d \mathbf{r}|^{2}}}=\frac{K\left(\boldsymbol{\rho}_{0} d \rho+\boldsymbol{\psi}_{0} \rho d v_{\overline{A B}}+\boldsymbol{\zeta}_{0} d \xi\right)}{\sqrt{(d \beta)^{2}+\left(\beta d v_{\overline{A B}}\right)^{2}+(K d \zeta)^{2}}} \\
& =\frac{\boldsymbol{\rho}_{0}+\boldsymbol{\psi}_{0} \beta\left(d v_{\overline{A B}} / d \beta\right)+\xi_{0} Z_{\overline{A B}}}{\sqrt{1+\beta^{2}\left(d v_{\overline{A B}} / d \beta\right)^{2}+Z_{\overline{A B}}^{2}}}=\frac{(d \mathbf{r} / d \beta)}{(d \sigma / d \beta)} \\
& \equiv \mathbf{n}_{0}=\frac{\boldsymbol{\rho}_{0} P_{\overline{A B}}+\boldsymbol{\psi}_{0}\left(Q_{\overline{A B}} / \beta\right)+\boldsymbol{\zeta}_{0} Z_{\overline{A B}}}{\sqrt{P_{\overline{A B}}^{2}+\left(Q_{\overline{A B}} / \beta\right)^{2}+Z_{\overline{A B}}^{2}}} \\
& =\frac{\boldsymbol{\rho}_{0}+\boldsymbol{\psi}_{0}\left(Q_{\overline{A B}} / \beta P_{\overline{A B}}\right)+\xi_{0}\left(Z_{\overline{A B}} / P_{\overline{A B}}\right)}{\sqrt{1+\left(Q_{\overline{A B}} / \beta P_{\overline{A B}}\right)^{2}+\left(Z_{\overline{A B}} / P_{\overline{A B}}\right)^{2}}} .
\end{aligned}
$$

Равенство $\beta\left(d v_{\overline{A B}} / d \beta\right)=\left(Q_{\overline{A B}} / \beta P_{\overline{A B}}\right)$ проекций векторов $d \mathbf{r} / d \sigma$ и $\mathbf{n}_{0}$ на направление $\boldsymbol{\psi}_{0}$ позволяет найти уравнения для элемента длины дуги $f$-траектории $\overline{A B}$ в виде

$$
\begin{aligned}
& \frac{d \sigma_{\overline{A B}}}{d \beta} \cong \frac{\mp 1}{K} \sqrt{1+\left(Q_{A B} / \beta P_{A B}\right)^{2}+\left(Z_{A B} / P_{A B}\right)^{2}} \\
& \equiv \frac{\sqrt{\left(1+Q_{A B}\right)^{2}+\left[\left(n^{2}-1+\bar{\alpha} \beta^{2}\right) /\left(1-\beta^{2}\right)\right]}}{\mp K\left|P_{A B}\left(Q_{A B}, Z_{A B}, \beta\right)\right|} .
\end{aligned}
$$


Длины $\sum_{\overline{A B}}$ и $\sum_{\overline{B A}}$ дуг „встречных“ $f$-траекторий на интервале $\left(\beta_{\bar{A}}, \beta_{\bar{B}}\right)$ вычисляются по правилам (21). В диэлектрике $\sum_{\overline{A B}} \cong 4.519 \mathrm{~cm}$ оказывается примерно на $2 \%$ больше $\sum_{\overline{B A}} \cong 4.425 \mathrm{~cm}$, при этом $\sum_{\overline{A B}}$ на $1 \%$ больше $S_{\overline{A B}}$ (длины дуги $R$-траектории $\overline{A B}$ ), а $\sum_{\overline{B A}}$, напротив, на $1 \%$ меньше $S_{\overline{B A}}$.

В вакууме $\left(\hat{n}^{2}=1\right)$ полная длина дуги $\hat{\sum}_{\overline{A B}} \cong 4.569 \mathrm{~cm} \quad f$-траектории $\overline{A B}$ на $4 \%$ больше полной длины дуги $\hat{\sum} \overline{B A} \cong 4.387 \mathrm{~cm}$ „встречной“ $f$-траектории $\overline{B A}$ и на $1 \%$ больше полной длины дуги $\hat{S}_{\overline{A B}} \cong 4.470 \mathrm{~cm}$ луча $\overline{A B}$. Напротив, $\hat{\sum}_{\overline{B A}}$ меньше длины дуги $\hat{S}_{\overline{B A}} \cong 4.468 \mathrm{~cm}$ луча $\overline{B A}$ на $1.8 \%$. (В ИСО $f$ - и $R$-траектории совпадают.)

Таким образом, в ВСО из-за неоднородности пространства длины дуг „встречных“ $f$-траекторий изменяются сильнее, чем длины дуг „встречных“ лучей. Как и в случае „встречных“ $R$-траекторий, длина дуги $f$-траектории $\overline{A B}$, имеющей положительную проекцию орта $\mathbf{n}_{0}$ на направление $\boldsymbol{\psi}_{0}$, оказывается больше длины дуги $f$-траектории $\overline{B A}$. При этом разница длин дуг „Встречных“ $f$-траекторий почти в 2 раза больше, чем аналогичный параметр „встречных“ $R$-траекторий.

\section{8. Оптическая длина пути и средние коэффицциенты преломления}

Уравнения для элемента оптической длины на пути $\overline{A B}$

$$
\begin{aligned}
& \frac{d \phi_{\overline{A B}}}{d \beta}=\frac{\mp(1 / K)}{\left|P_{\overline{A B}}\left(Q_{\overline{A B}}, Z_{\overline{A B}}, \beta\right)\right|} \\
& \times\left\{1+Q_{\overline{A B}}+\left[\left(n^{2}-1+\bar{\alpha} \beta^{2}\right) /\left(1-\beta^{2}\right)\right]\right\}
\end{aligned}
$$

получаются непосредственно из (15). Полные оптические длины $\Phi_{\overline{A B}}$ и $\Phi_{\overline{B A}}$ траекторий $\overline{A B}$ и $\overline{B A}$ вычисляются совершенно так же, как длины дуг $S_{\overline{A B}}$ и $S_{\overline{B A}}$. В диэлектрике оптические длины $\Phi_{\overline{A B}} \cong 7.828 \mathrm{~cm}$ и $\Phi_{\overline{B A}} \cong 7.665 \mathrm{~cm}$ „встречных“ лучей различаются примерно на $2 \%$, и также на $2 \%$ различаются средние значения коэффициентов преломления $n_{\overline{A B}} \equiv\left(\Phi_{\overline{A B}} / S_{\overline{A B}}\right) \cong 1.750$ и $\quad n_{\overline{B A}} \equiv\left(\Phi_{\overline{B A}} / S_{\overline{B A}}\right) \cong 1.715$, которые в недиспергирующей среде определяют средние скорости $\left(c / n_{1,2}^{R}\right)$ светового импульса (групповые скорости) на $R$-траекториях $\overline{A B}$ и $\overline{B A}$. В вакууме полные оптические длины $\hat{\Phi}_{\overline{A B}}=4.550 \mathrm{~cm}$ и $\hat{\Phi}_{\overline{B A}}=4.387 \mathrm{~cm}$ „встречных“ $R$-траекторий и средние значения коэффициентов преломления $\quad \hat{n}_{\overline{A B}}^{R} \equiv\left(\hat{\Phi}_{\overline{A B}} / \hat{S}_{\overline{A B}}\right) \cong 1.018$ и $\quad \hat{n} \frac{R}{B A} \equiv\left(\hat{\Phi}_{\overline{B A}} / \hat{S}_{\overline{B A}}\right) \cong 0.982$ различаются примерно на $3.7 \%$. В ИСО оптические длины „встречных“ $R$-траекторий одинаковы: $\left(n \frac{R}{A B}\right)_{K=0}=\left(n \frac{R}{B A}\right)_{K=0}=3^{1 / 2}$ в диэлектрике и $\left(\hat{n} \frac{R}{A B}\right)_{K=0}=\left(\hat{n} \frac{R}{B A}\right)_{K=0}=1$ в вакууме.

Неоднородность пространства и увлечение света движущейся средой как независимые физические механизмы изменяют оптические длины „встречных“ лучей, противодействуя друг другу. Наибольшая разница „встречных“ оптических длин имеет место в вакууме. Увлечение света движущейся средой уменьшает ее почти в 2 раза.

Оптическую длину $\Phi_{\overline{A B}}$ можно считать также оптической длиной $f$-траектории $\overline{A B}$ и ввести средний показатель преломления $\bar{n}_{\overline{A B}}^{f} \equiv\left(\Phi_{\overline{A B}} / \sum_{\overline{A B}}\right)$. В анизотропной недиспергирующей среде отношение $\left(c / n \frac{f}{A B}\right)$ может иметь смысл средней фазовой скорости на $f$-траектории $\overline{A B}$. Поскольку длина $\sum_{\overline{A B}}$ дуги $f$-траектории $\overline{A B}$ больше длины $S_{\overline{A B}}$ дуги луча $\overline{A B}$ на $1 \%$, то также на $1 \%$ средний коэффициент преломления $n \frac{R}{A B} \equiv\left(\Phi_{\overline{A B}} / S_{\overline{A B}}\right)$ больше $n_{\overline{A B}}^{f} \equiv\left(\Phi_{\overline{A B}} / \sum_{\overline{A B}}\right) \cong 1.732$. На „встречном“ оптическом пути $\overline{B A}$ средние значения коэффициентов преломления $n_{\overline{B A}}^{R} \equiv\left(\Phi_{\overline{B A}} / S_{\overline{B A}}\right) \cong 1.723$ и $n_{\overline{B A}}^{f} \equiv\left(\Phi_{\overline{B A}} / \sum_{\overline{B A}}\right) \cong 1.732 \cong n_{\overline{A B}}^{f}$ различаются примерно на $0.5 \%$, причем в отличие от предыдущего случая на траекториях $\overline{B A}$ справедливо $n_{\overline{B A}}^{R}<n_{\overline{B A}}^{f}$.

\section{9. Ретрансляционная сеть и двухзеркальный интерферометр Фабри-Перо в ВСО}

При проектировании в вакууме ретрансляционной сети $\bar{A} \leftrightarrow \bar{B}$ следует предусмотреть возможность установки ретранслятора, принимающего сигнал из пункта $\bar{A}$, в пункте $\bar{B}_{R}$ и отправку отраженного импульса по „встречной“ $R$-траектории $\bar{B}_{R} \bar{A}$. При малой разнице координат пунктов $\bar{B}$ и $\bar{B}_{R}\left(\delta \hat{\psi}_{\overline{A B}} \approx 2.712 \times 10^{-3}\right)$ параметры зависимостей $\hat{\psi}_{\overline{B A}}(\beta)$ и $\hat{\psi}_{\overline{B A}(\beta)}$ будут очень близки. Поэтому отраженный импульс следует ожидать в пункте $\bar{A}_{R R}$, координата $\psi_{\bar{A}_{R R}}$ которого будет смещена относительно координаты $\psi_{\bar{A}}$ пункта $\bar{A}$ на величину $\delta \hat{\psi}_{\overline{A A}_{R R}} \approx \delta \hat{\psi}_{\overline{B A}}-\delta \hat{\psi}_{\overline{A B}} \approx 0.416 \cdot 10^{-3}$. Формально этот теоретический результат означает отсутствие в ВСО замкнутого оптического пути. Однако в рассматриваемом примере расстояния $L_{\overline{A A}_{R R}} \approx 0.0083 \mathrm{~mm}$ между точками $\bar{A}$ и $\bar{A}_{R R}$, а также $L_{\overline{B B}_{R R}} \approx 0.11 \mathrm{~mm}$ между $\bar{B}$ и $\bar{B}_{R}$, в десятки раз меньшие ширины лазерного пучка, вероятно, не изменят характеристики оптической ретрансляционной сети, при построении которой неоднородность пространства не учитывалась. Но в сети, где размеры треугольника $A O B$ порядка нескольких километров, а угловая скорость вращения (для сохранения принципа подобия) уменьшена в $10^{5}$ раз, неконтролируемое изменение трассы $\left(L_{\overline{A A}_{R R}}\right)_{2} \approx 83 \mathrm{~cm}$ будет уже серьезным препятствием для создания замкнутого оптического пути.

Из (23) следует, что в пунктах $\bar{A}$ и $\bar{B}$ орты $\mathbf{s}_{0}\left(\beta_{\bar{S}}, \pm A_{\overline{A B}}, 0\right)$ „встречных“ $R$-траекторий образуют углы, отличающиеся от $\pi$ на $\Theta \frac{R}{S} \neq 0$. Для создания замкнутого оптического пути $\bar{A} \leftrightarrow \bar{B}$ (в случаях, когда можно пренебречь неконтролируемым изменением трассы) в каждом конечном пункте $\bar{S}$ необходимо иметь рефлектор, способный отражать приходящую волну под строго определенным углом между „встречными“ 
$R$-траекториями. Уголковые отражатели (ретрорефлекторы) и нелинейные зеркала с обращением волнового фронта приходящего излучения, которые отражают каждую падающую волну с волновым вектором $\mathbf{k}_{q}$ в направлении $\left(-\mathbf{k}_{q}\right)$, не могут решить такую техническую задачу. Этот очевидный вывод совпадает с результатом работы [18], полученным с помощью решения в приближении геометрической оптики уравнений для изотропных геодезических волн в вакууме. Концептуально проблему можно решить с помощью адаптируемых к частоте вращения $\Omega$ плоских зеркал, у которых нормали к отражающим поверхностям $\mathbf{n}_{\bar{S}}^{0}$ должны образовывать угол $\pi+\Theta_{\bar{S}}^{R} / 2$ с ортом $\mathbf{s}_{0}\left(\beta_{\bar{S}}, \pm Q_{\overline{A B}}, 0\right)$ падающего на зеркало излучения.

Для осуществления в ВСО генерации лазера, имеющего резонансную систему типа двухзеркального резонатора Фабри-Перо, необходимо иметь замкнутый оптический путь $\bar{A} \leftrightarrow \bar{B}$, созданный парой „встречных“ $f$-траекторий. В соответствии с (14) на зеркалах $\bar{A}$ и $\bar{B}$ орты $\mathbf{n}_{0}\left(\beta_{\bar{S}}, \pm Q_{\overline{A B}}, 0\right)$ „встречных“ $f$-траекторий образуют углы $\pi+\Theta_{\bar{S}}^{f}$. Формирование такого замкнутого оптического пути возможно, если использовать адаптируемые к частоте вращения плоские зеркала, нормали которых $\mathbf{n}_{\bar{S}}^{0}$ должны иметь углы $\pi+\Theta_{\overline{A B}}^{f} / 2$ с ортами $\mathbf{n}_{0}\left(\beta_{\bar{S}}, \pm Q_{\bar{A} \bar{B}}, 0\right)$ приходящих волновых фронтов.

Следует заметить, что нарушение условий генерации излучения в лазере с резонатором Фабри-Перо может произойти из-за смещения $f$-траекторий в поперечном направлении от оси активного элемента. В случае, когда отклонение траектории от оси активного элемента (при $\beta \approx \beta_{\overline{A B}}, \beta_{\overline{B A}}$ ) окажется сопоставимым с радиусом его поперечного сечения, из-за влияния границы элемента должны измениться структура поля генерируемой моды и параметры (в частности, частота) лазерного излучения.

\section{0. Неоднородность пространства в ВСО и эффект Саньяка}

Ocобое значение для изучения эффекта Саньяка имеют круговые $\left(\rho=\right.$ const $\left._{\rho}\right) f$-траектории в плоскости $\xi=$ const $_{\xi}$ вращающегося диэлектрика, поскольку они могут рассматриваться как элементы одномерной модели моды кольцевого резонатора, образуемой парой встречных бегущих волн. Такие волновые фронты формируются эйконалами $\Phi(\beta, Q)$, которые с учетом $(\partial \Phi / \partial \rho)=(\partial \Phi / \partial \xi)=0$ и $(\partial \Phi / \partial \psi)=(Q / K)$ удовлетворяют уравнению (8) в виде

$$
-(Q / \beta)^{2}+\left[\left(\bar{n}^{2}-1\right) /\left(1-\beta^{2}\right)\right]+(1+Q)^{2}=0 .
$$

Корням

$$
Q^{ \pm}=\beta\left[\left(\beta \pm \sqrt{\bar{n}^{2}}\right) /\left(1-\beta^{2}\right)\right]
$$

квадратного уравнения (38) соответствуют эйконалы

$$
\Phi^{ \pm}= \pm \rho\left[\left(\beta \pm \sqrt{\bar{n}^{2}}\right) /\left(1-\beta^{2}\right)\right]\left(\psi^{ \pm}-\psi_{0}^{ \pm}\right),
$$

по-разному изменяющиеся на участках $\rho\left|d \psi^{ \pm}\right|$собственных фазовых траекторий:

$$
\left(\left|d \Phi^{+}\right| /\left|d \psi^{+}\right|\right) /\left(\left|d \Phi^{-}\right| /\left|d \psi^{-}\right|\right)=\left(\sqrt{\bar{n}^{2}}+\beta\right) /\left(\sqrt{\bar{n}^{2}}-\beta\right) .
$$

Из (41) видно, что по направлению вращения $\boldsymbol{\psi}_{0}$ эйконал $\Phi^{+}$на длине $\rho|d \psi|=\rho\left|d \psi^{+}\right|$растет быстрее, чем эйконал $\Phi^{-}$на такой же длине $\rho|d \psi|=\rho\left|d \psi^{-}\right|$по встречному направлению $\left(-\boldsymbol{\psi}_{0}\right)$. Это различие наиболее существенно в вакууме и практически исчезает при $\sqrt{\bar{n}^{2}} \gg \beta$. Следовательно, (41) характеризует различие в свойствах координат $\psi^{ \pm}$и тем самым неоднородность координатного пространства в ВСО, которая по своей сути является физической причиной эффекта Саньяка.

Условие для определения собственных частот $\omega_{q}^{ \pm}$ кольцевого резонатора в приближении ГО заключается в том, чтобы на полной (замкнутой) длине дуги $2 \pi \rho$ собственной (кольцевой) фазовой траектории каждого эйконала $\Phi_{ \pm}\left(\rho, \sqrt{\bar{n}^{2}}, \psi^{ \pm}=2 \pi\right)$ укладывалось целое число $q$ длин волн $\lambda_{q}^{ \pm}=\left(c 2 \pi / \omega_{q}^{ \pm}\right)$:

$$
\left(\omega_{q}^{ \pm} / c\right)\left\{ \pm \rho\left[\left(\beta \pm \sqrt{\bar{n}^{2}}\right) /\left(1-\beta^{2}\right)\right] 2 \pi\right\}= \pm 2 \pi q .
$$

Из (42) находятся собственные частоты кольцевого резонатора по формуле

$$
\omega_{q}^{ \pm}=\left[q c\left(1-\beta^{2}\right) / \rho\left(\sqrt{\bar{n}^{2}} \pm \beta\right)\right]
$$

которая совпадает с формулой (36) в работе [12]. В [12] показано, что в этом случае в эффекте Саньяка не учитывается вклад, который вносит в дисперсионные свойства вращающейся среды неоднородность свободного пространства по координате $\rho$, обусловленная вращением.

\section{1. Выводы}

Вращающийся твердотельный однородный изотропный диэлектрик без дисперсии в сопровождающей вращение системе отсчета оказывается неоднородной анизотропной средой из-за воздействия двух конкурирующих физических механизмов: обусловленной вращением неоднородности свободного пространства и увлечения света движущейся средой.

В приближении ГО в таком вращающемся диэлектрике получены уравнение эйконала и соответствующая ему система обыкновенных дифференциальных уравнений в характеристической форме. Решениями уравнений при расчетных параметрах, определенных с помощью первых интегралов системы, являются пары „встречных“ $R$-траекторий лучей и $f$-траекторий волновых фронтов. Получена формула для интенсивности светового импульса, распространяющегося по произвольной $R$-траектории.

„Встречные g“ траектории обоих типов не имеют общих точек и смещены в противоположные стороны от 
прямой линии между конечными пунктами. Их структурные параметры (минимальное расстояние до оси вращения, длина дуги, область определения по азимутальной координате $\psi$, оптическая длина и др.) изменяются под воздействием обоих физических механизмов, зависящих от скорости вращения.

Замкнутые оптические пути в ВСО для ретрансляционной сети и для двухзеркального резонатора ФабриПеро в генерирующем лазере можно создать с помощью двух типов адаптируемых к частоте вращения плоских зеркал, у которых нормали к отражающим поверхностям должны иметь определенные разные углы с ортами лучевых векторов и волновых фронтов приходящего на рефлектор излучения.

Эффект Саньяка является следствием неоднородности (деформации) свободного пространства по азимутальной координате, а его величина - результат конкурирующего воздействия обоих физических механизмов.

\section{Благодарности}

Авторы выражают благодарность профессору А.В. Кудрину за интерес к работе и ценные замечания.

\section{Конфликт интересов}

Конфликт интересов отсутствует.

\section{Список литературы}

[1] Розанов Н.Н., Сочилин Г.Б. // УФН. 2006. Т. 176. № 4. C. 221. doi 10.3367/UFNr.0176.200604f.0421

[2] Розанов Н.Н., Сочилин Г.Б. // Оптический журн. 2009. T. 76. № 4. C. 86.

[3] Sagnac M.G. // Compt. Rend. 1913. V. 157. N 17. P. 708.

[4] Малыкин Г.Б. // УФН. 1997. Т. 167. № 3. С. 347. doi 10.3367/UFNr.0167.199703i.0347

[5] Малыкин Г.Б. // УФН. 2000. Т. 170. № 12. С. 1325. doi 10.3367/UFNr.0170.200012c.1325

[6] Андронова И.А., Малыкин Г.Б. // УФН. 2002. Т. 172. № 8. C. 849. doi 10.3367/UFNr.0172.200208a.0849

[7] Малыкин Г.Б. // УФН. 2002. Т. 172. № 8. С. 969. doi 10.3367/UFNr.0172.200208k.0969

[8] Малыкин Г.Б.// УФН. 2014. Т. 184. № 7. С. 775. doi 10.3367/UFNr.0184.201407g.0775

[9] Yildiz A., Tang C.H. // Phys. Rev. 1966. V. 146. N 4. P. 947. doi 10.1103/PhysRev.146.947

[10] Post E.J. // Rev. Mod. Phys. 1967. V. 39. N 2. P. 475. doi 10.1103/RevModPhys.39.475

[11] Anderson J.L., Ryon J.W. // Phys. Rev. 1969. V. 181. N 5. P. 1765. doi 10.1103/PhysRev.181.1765

[12] Миловский Н.Д. // Опт. и спектр. 2017. Т. 123. № 4. C. 633 . doi 10.7868/S0030403417100142); Milovskii N.D. // Opt. Spectrosc. 2017. V. 123. N 4. P. 642. doi 10.1134/S0030400X17100149

[13] Ландау Л.Д., Лифиии, Е.М. Гидродинамика. М.: Наука, 1986. $736 \mathrm{c}$

[14] Степанов В.В. Курс дифференциальных уравнений. М.: ГИФМЛ, 1958. 468 с.
[15] Курант Р. Уравнения с частными производными. М.: Мир, 1964. $830 \mathrm{c}$.

[16] Кравцов Ю.А., Орлов Ю.И. Геометрическая оптика неоднородных сред. М.: Наука, 1980. 304 с.

[17] Born M.A., Wolf E. Principles of Optics. Londjn: Pergamon Press, 1964. 896 р; Борн М., Вольø Э. Основы оптики. М.: ГИФМЛ, 1970. $856 \mathrm{c.}$

[18] Денисов М.М., Кравцов Н.В., Кривченков И.В. // Письма в ЖЭТФ. 2007. Т. $85 . \quad$ В. $8 . \quad$ С. 498. doi $10.1134 / \mathrm{S} 0021364007080140$ 